\title{
Sources médicinales et thermalisme dans le bassin de l'Ebre. Les problèmes de la documentation antique
}

\author{
NICOLE DUPRÉ *
}

On utilise indifféremment les mots «thermes» et «bains» pour désigner les établissements romains, qu'ils aient eu une fonction médicale (comme les thermes d'Apollon à Vicarello) ou seulement hygiénique (ainsi les thermes de Caracalla à Rome). Cette confusion se retrouve dans les noms de villes d'eaux (Barbotan-les-Thermes et Amélie-les Bains par exemple), dont l'origine est le plus souvent antique.

L'ambiguité vient de la mise au point par les Romains d'un système de chauffage permettant de recrèer artificiellement dans les bains la température souvent élevée des sources minéro-médicinales: d'où l'emploi, à côté de «balneae», de «thermae» qui ne renseigne d'ailleurs pas sur la nature de l'établissement: en effet l'eau d'une piscine ne devient pas bénéfique parce que chauffée par hypocauste, inversement toutes les sources curatives ne sourdent pas chaudes. Comme c'est malgré tout le cas de la plupart d'entre elles, «thermal» a fini par se référer au seul domaine médical et «thermes» n'est plus utilisé de nos jours pour désigner les bains à simple caractère ludique. Le clivage s'est dèfinitivement imposé depuis que le thermalisme a perdu le caractère touristique voire mondain qu'il avait encore au $X I x^{e}$ siècle: aujourd'hui faire une cure ne relève plus que de la stricte therrapeutique.

II serait souhaitable d'appliquer une telle distinction à l'époque romaine, c'est à dire d'appeler "bains" les établissements où -même chaude-l'eau est dépourvue de vertu curative et den'utiliser «thermes» que

* CNRS. Paris. 
lorsqu'elle a une fonction médicinale avérée: ainsi les thermes de Caracalla sont'ils en fait des bains et Amélie-les-Bains une ville thermale malgré son nom. Mais la documentation ne permet pas toujours de reconnaître aussi aisément la nature — voire l'existence- des sites antiques, comme on peut le constater par l'exemple du bassin de l'Ebre.

Par rapport au Noroeste à dominante celtique ou au Noreste fortement romanisé, le nord de l'Hispanie apparaît comme une zone très composite. Sur le plan ethnique, elle était habitée par des peuples d'origines différentes mais acculturés entre eux depuis des siècles à l'arrivée des Romains. Sur le plan historique, le secteur cantabrique du bassin ne fut conquis qu'à l'époque d'Auguste alors que ses parties orientale et centrale l'avaient été dès la République. Enfin les dépressions de l'Ebre ou de ses affluents connurent une romanisation plus précoce et plus intense que les reliefs périphériques. L'ampleur du bassin (un sixième de l'Espagne) explique une telle diversité, qui a déterminé le découpage administratif antique: sous l'Empire en effet, le cours supérieur de l'Ebre faisait partie du conventus de Clunia, le cours moyen de celui de Caesaraugusta et le cours inférieur de celui de Tarraco.

Aujourd'hui l'ensemble hydrographique se trouve morcelé entre plusieurs régions et de multiples provinces, ce qui entraîne la dispersion des renseignements et ne facilite pas une vision d'ensemble du sujet. Néanmoins il nous a semblé intéressant d'étudier la documentation à l'échelle du bassin, en raison précisément de son hétérogénéité: on pourra ainsi comparer les formes qu'y prend le thermalisme antique avec celles de zones limitrophes plus homegènes ou mieux connues.

En effet, à la différence aussi du Noroeste ou du Noreste, le nord de l'Hispanie n'avait pas encore fait l'objet d'une recherche particulière en ce domaine: nous avons pu le constater l'an dernier en présentant un premier état de la question ${ }^{1}$ au colloque d'Aix-les-Bains, qui fut à l'origine de la table-ronde de Madrid. Il y a pourtant dans le bassin de l'Ebre de très nombreuses sources minéro-médicinales, dont la plupart sont encore en service de nos jours et dont on sait que certaines l'étaient déjà dans l'Antiquité. Mais, comme les textes romains ne mentionnent ni leur exploitation ni même leur existence, les données que nous possédons sont indirectes et concernent des sites dont le caractère thermal n'est pas tou-

\footnotetext{
1 Dupre, N., \& PEREX, M. J., «Thermalisme et religion dans le nord de l'Hispania», Les eaux thermales et les cultes des eaux en Gaule et dans les provinces voisines (Aix-lesBains, 1990). Tours/Turin 1992, págs. 151-168 et 466-468.
} 
jours facile à établir ${ }^{2}$. Aussi les étudierons-nous en indiquant, pour chacun d'eux, la nature et la valeur de la documentation disponible.

Une activite thermale antique n'est attestée à ce jour qu'en quatre lieux ${ }^{3}$ du bassin de l'Ebre [fig. 1] à Alhama de Aragón (par des textes, des inscriptions et des restes archéologiques), à Tiermas (par l'archéologie et l'épigraphie), à Fitero et Panticosa (par la seule archéologie).

Il existe des allusions au thermalisme antique dans les documents routiers romains, au premier rang desquels la carte dite de Peutinger qui distingue les villes d'eau par une vignette spéciale. Bien que ce témoignage graphique fasse défaut pour l'Hispania (qui a disparu avec le début du volumen), des villes appelées «Aquae» apparaissent parmi les étapes antiques: ainsi dans l'Itinéraire d'Antonin, où la plupart des Aquae appartiennent au nord-ouest de la Péninsule Ibérique mais où figurent aussi deux noms de ce type pour le nord-est et un pour le nord.

Si la localisation des thermes d'Aquae Calidae à Caldas de Montbuy ou à Caldas de Malavella n'est pas évidente ${ }^{4}$, celle d'Aquae Bilbilitanorum à Alhama de Aragón n'a jamais fait problème: il est vrai que l'Itinéraire d'Antonin nomme cette mansio par deux fois (en 437, 2 et 438, 14) sur la via Emerita-Caesaraugusta, à vingt-quatre milles au sud de Bilbilis, cité parfaitement identifiée près de Calatayud et où fut découverte l'inscription funéraire d'un Aquensis ${ }^{5}$.

Du mot latin au mot arabe (Alhama) présent dans le nom actuel de la ville — qui s'appela même Baños de Alhama ${ }^{6}$ — s'est maintenue une allu-

\footnotetext{
${ }^{2}$ Comme on peut également le voir dans l'étude parallèle de M. J. Peréx \& M. Unzu consacrée aux habitats préromains du territoire vascon.

${ }^{3} \mathrm{Ne}$ figurent pas sur la carte les multiples établissements et inscriptions du Val d'Aran, situé dans le bassin de la Garonne et donc rattachè à la Gaule romaine, alors qu'il dépend aujourd'hui de la province espagnole de leérida. Bibliographie commune aux quatres sites antiques: MoRA, G., "Las termas romanas en Hispania", AEA, 54, 1981, págs. 37-90. DIEZ DE VELASCO, F., Balnearios y divinidades de las aguas termales en la Peninsula lbérica en época romana. Madrid 1987.

${ }^{4}$ Voir MAYER, M., \& RODA. I., «L'epigrafia romana a Catalunya, estat de la qüestió i darreres novetats', Fonaments, 5, 1983, págs. 182-185.

${ }^{5}$ CIL 2, 3022: L CORNELIUS / PHILOMUSI / L SAMIUS / AQUENSIS / HSE ( $1^{\text {ere }}$ moitié du $\mathrm{I}^{\mathrm{er}}$ siècle de notre ère). Voir MARTIN BUENO, M. A., «Una conocida inscripción romana de Bilbilis", Estudios del Seminario de Prehistoria, Arqueología e Historia antigua de la Facultad de Filosofía y Letras de Zaragoza, 2, 1973, págs. 151-154.

${ }^{6}$ Madoz, J., Diccionario geográfico-estadistico de España y sus provincias de Ultramar. Madrid 1845-1850, t. 1, pág. 584: «... El nombre Baños de Alhama es uno de tantos pleonasmos, que ha producido en la nomenclatura geográfica la mezcla de idiomas; las voces Aquae, Alhama y Baños, expresan una misma idea...».
} 


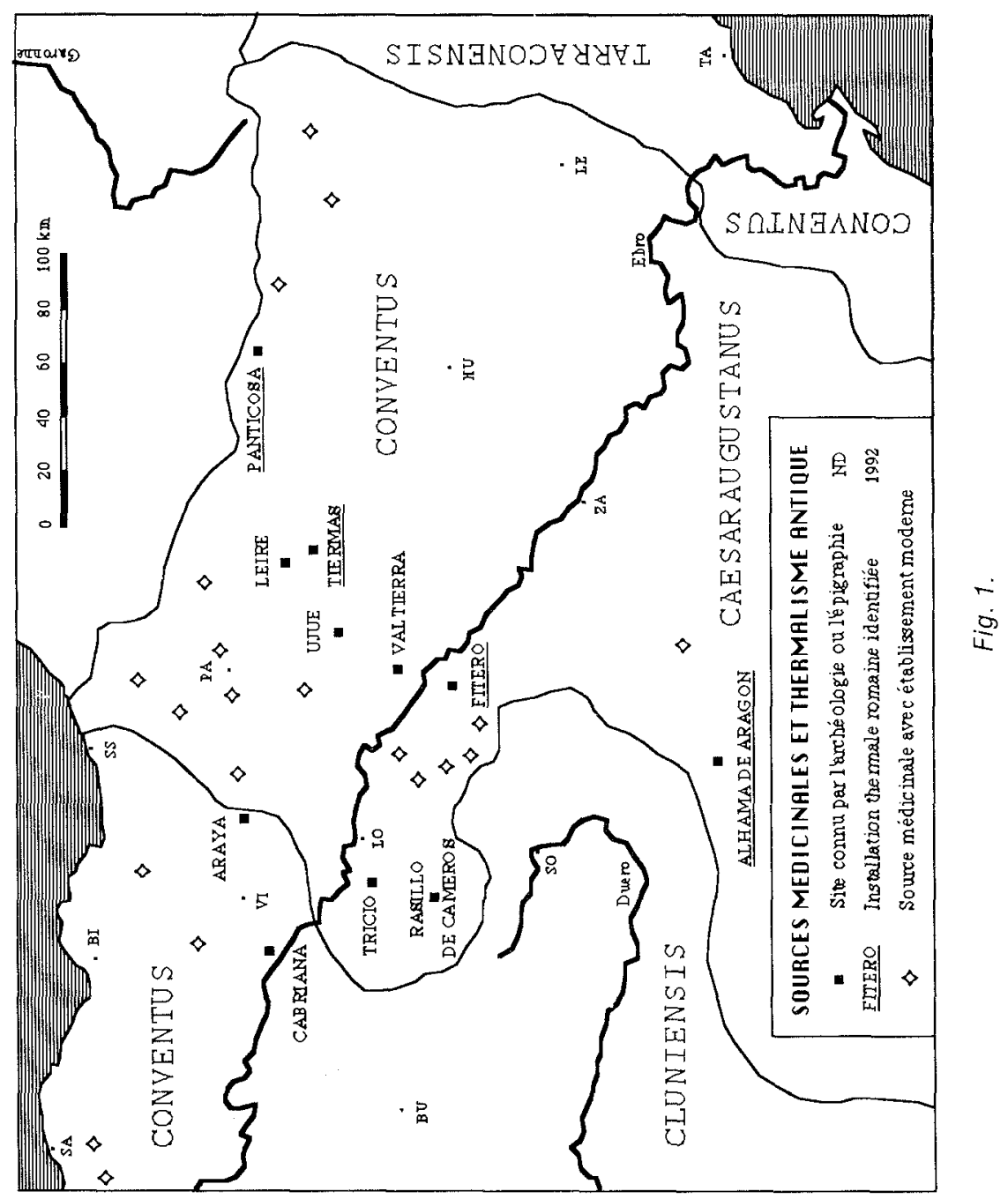


sion directe aux eaux thermales du lieu, en l'occurence les sources (bicarbonatées surtout, mais aussi calciques et radioactives) qui jaillissent à $32 ' 37^{\circ} \mathrm{C}$ : leur utilisation thérapeutique nous est signalée dès le MoyenAge mais on peut la faire remonter jusqu'à l'Antiquité.

En effet, bien que les structures romaines ne soient plus visibles de nos jours ${ }^{7}$, nous avons la preuve de leur existence par la description qu'en firent au $\mathrm{XIX}^{\mathrm{e}}$ siècle les érudits Ceán Bermúdez et Madoz ${ }^{8}$. Selon ce dernier elles formaient la partie la plus ancienne des baños viejos, qui sont mentionnés en 1112 sur la rive du rio Jalón et qu'on appelle ainsi par opposition aux baños nuevos d'Alhama de Aragón construits en 1827.

De même a-t-on découvert à proximité de la ville une inscription ${ }^{9}$ qui se rapporte certainement aux thermes antiques et invoque la divinite (ou une divinité) protectrice du site: celui-ci devait déjà fonctionner à l'époque préromaine, à en juger par la formule ${ }^{10}$ syncrétrique deo tutel désignant la figure locale. S'agit-il ${ }^{11}$ du Congedus évoqué par Martial ${ }^{12}$, dont l'eau tiède est associée par le poète aux Nymphes qui ont très souvent partagé ou repris le rôle des divinités thermales indigènes? La mention du Jalón par l'auteur porte du moins à croire que le texte concerne bien Aquae Birbilitanorum, dont Congedus pourrait aussi représenter l'ancien nom celtibère ${ }^{13}$.

Si Alhama de Aragòn est le site du bassin de l'Ebre pour lequel nous disposons du maximum de renseignements, paradoxalement c'est aussi celui dont nous connaissons le plus mal les aménagements antiques, l'absence de fouilles interdisant à l'heure actuelle de préciser le plan et la chronologie des constructions, du moins à l'époque romaine.

Auparavant, les populations indigènes avaient pu se contenter de mettre à profit le fait que la source jaillit d'une voûte calcaire et se déverse dans le Jalón voisin par des fissures qu'il suffit de boucher pour remplir les cavités de la roche: du Moyen-Age à l'entrée en service des baños

\footnotetext{
7 Lostal Pros, J., «Arqueologia del Aragón romano», Caesaraugusta, 47-48, 1979, págs. 233-296.

${ }^{8}$ Cean Bermudez, A., Sumario de las antigüedades romanas que hay en España. Madrid 1832, pág. 125. MADOz. J., op. cit., t. 1, p. 584-585.

' CIL 2, 3021: DEO TUTEL / GENIO LOCI.

${ }^{10}$ On retrouve ce rapprochement entre Tutela et une figure masculine dans la dédicace au Genio Tutelae Horreorum de Caesaraugusta (C/L 2, 2991). Voir PENA, M. J., «El culto a Tutela en Hispania», MHA, 5, 1981, págs. 73-87.

${ }^{11}$ Schulten, A., Fontes Hispaniae Antiquae. Madrid, t. 8, 1959, pág. 253.

${ }^{12}$ Epigrammes, 1, 49, 9-12: «Tepidi natabis lene Congedi vadum / Mollesque Nympharum lacus / Quibus remissum corpus adstrigi brevi / Salone, qui ferrurn gelat».

13 Lostal. Prost, J., op. cit., pág. 264.
} 
nuevos, les deux bains réservés aux hommes et aux femmes ont ainsi fonctionné sans canalisations ni baignoires, en utilisant simplement la configuration naturelle du lieu. En revanche, le nom d'Aquae Bilbilitanorum et sa position sur la route de Lusitanie, à proximité de Bilbilis (municipe de droit romain fort prospère sous l'Empire), suggèrent un établissement thermal «à la romaine», disposant d'adductions d'eau et de piscines ${ }^{14}$ semblables à celles qu'on a mises au jour en d'autres points du nord de l'Hispanie. Et, comme Aquae Bilbilitanorum fut crée sur un site thermal qui demeure aujourd'hui le plus important d'Aragon, on peut se faire une idée de l'ampleur de ses installations antiques en voyant les structures conservées d'ensembles plus modestes.

A notre connaissance, aucun texte ancien ne mentionne les trois autres sites thermaux du bassin de l'Ebre don l'existence est vérifiée pour la période romaine. Mais que Tiermas, Fitero ou Panticosa soient absents de l'Itinéraire d'Antonin n'empêche pas qu'ils aient pu servir de relais d'étape ${ }^{15}$ : on sait que ce document ne recense pas la totalité de la voirie romaine et qu'il ne peut nous renseigner sur celle du Haut-Empire, où existaient déjà des installations thermales. Or, en dépit de leur isolement apparent, les trois lieux se trouvaient sur des axes de ce réseau secondaire que les prospections ont récemment révélé en Aragon et en Navarre ${ }^{16}$. Et la relation au système routier a dû jouer un rôle essentiel dans le maintien -ou l'essor- de certains sites thermaux à la période romaine, soit que la présence d'une eau médicinale ait pérennisé sous l'Empire le tracé d'un chemin indigène, soit que la création d'un nouvel itinéraire ait favorisé la fréquentation de la source la plus proche parmi celles aux propriétés identiques et déjà connues des populations.

Des voies locales - moins importantes qu'à Aquae bilbilitanorumdesservaient Tiermas, Fitero et Panticosa, qui eux aussi paraissent avoir été des sites ruraux (mais il conviendrait de le vérifier par des fouilles): rien de surprenant donc à ce qu'on n'ait conservé aucun texte antique à propos de ces établissements bien que l'archéologie prouve leur existen-

${ }^{14} \mathrm{Si}$ les mots vadum et lacus ainsi que la mention du Salo par Martial (né à Bilbilis) évoquent des installations d'origine naturelle (ce qui s'appliquerait bien à Alhama de Aragón), les activités décrites (bains tiède puis froid) renvoient aux tepidarium et frigidarium de l'ensemble balnéaire romain (thermal ou hygiénique).

${ }^{15}$ Un document royal de 1213 metionne le toponyme Aquis parmi les terres relevant de Tiermas. La bourgade avait été fondée en 1201 au nord-ouest de l'ensemble thermal aujourd'hui appelé Baños de Tiermas. Voir la bibliographie à la note 17.

${ }^{16}$ Magallon, M. A., La red viaria romana en Aragón. Zaragoza 1987. Peréx, M. J., Los Vascones. Pamplona 1986. 
ce, du moins pour l'Empire car nous ignorons s'ils étaient en activité sous la République ou à l'époque indigène.

Depuis 1959 le site de Tiermas gît sous les eaux du lac créé par le barrage de Yesa. Auparavant fonctionnait au lieu-dit Baños de Tiermas un ensemble thermal établi au pied de la sierra de Leire sur la rive droite du rio Aragón et qui exploitait une source (à dominante sulfurée mais également sodique et chlorurée) jaillissant à $33^{\circ} \mathrm{C}$. Ces thermes semblent avoir eu une certaine importance au Moyen-Age car c'est le seul endroit de l'actuelle province de Saragosse mentionné par le Guide des Pélerins (xII $^{e}$ siècle) sur le Camino Real allant vers Saint-Jacques de Compostelle ${ }^{17}$. A l'édifice thermal s'ajoutèrent un hospice (appelé domum de Termis dans un document de l'époque) et une bourgade royale (l'actuelle Tiermas) fondée en 1201. A la fin du siècle, un pont permettait de rejoindre le travé sud du Camino Real, qui reprenait (sur la rive gauche du río Aragón) celui de la route romaine -et sans doute préromaineJaca-Sangüesa: cet axe transversal mettait en relation les voies allant de Caesaraugusta vers Beneharno et Pompaelo.

Une piscine circulaire romaine - signalée depuis longtemps ${ }^{18}$ - était encore visible dans l'établissement moderne avant sa submersion, qui interdit désormais toute fouille en vue de retrouver les installations antiques. On ne connaît donc ni leur plan ni leur chronologie, seulement leur existence grâce à ce renseignement archéologique.

Sans doute peut-on y ajouter le témoignage épigraphique d'un autel votif en grès ${ }^{19}$, découvert au milieu du siècle à Leire où il avait été réutilisé dans une aile ancienne du monastère. Comme ce dernier ne se trouve qu'à quelques kilomètres au nord-ouest de Baños de Tiermas ${ }^{20}$, les antiquités provenant de la rive droite de l'Aragon furent longtemps rassemblées à Leire: elles formèrent, avec celles recueillies sur l'autre rive

${ }^{17}$ Contin, S., «Historia de Tiermas». Zaragoza, 16, 1964, págs. 9-62, repris dans Historia de Tiermas. Zaragoza 1967. Passinı, J., Guia del Camino de Santiago en Aragón. Zaragoza 1989. Lamban Montanes, j., «El Camino de Santiago, bajo las aguas del Pantano de Yesa», Suessetania (Ejea de los Caballeros, Zaragoza), 11, junio 1990, págs. 2-12.

18 Traggia, J., Aparato a la historia eclesiástica de Aragón. Madrid 1791-1792, t. 2, págs. 224-225. Cean Bermudez, op. cit., pág. 158. Lostal Pros, «Arqueologia del Aragón romano", Caesaraugusta, 41-42, 1977, págs. 17-18, qui ajoute: "También se descubrieron algunas monedas romanas de las cuales no se detalla nada más».

${ }^{19}$ QVINTVS / LICINIVS / [FIVSCVS / AQVILEGVS VARAIEN / SIS NIMPIS / VIVENS M / V S (ILER 614).

${ }^{20}$ Avant d'être rattaché —avec d'autres établissements religieux du Camino Real-au prieuré de Sainte-Christine du Somport par le pape Innocent Ill, l'hôpital Saint-Martin de Tiermas (domum de Termis) relevait du monastère navarrais de Leire, comme son voisin d'Artieda de Aragón (domum de Arteda). Lamban Montanes, J., op. cit., pág. 10. 
au monastère de Javier et plus en aval au monastère de la Oliva, une grande partie des collections actuelles du Museo de Navarra ${ }^{21}$.

C'est pour cette raison qu'on rattache l'inscription à l'ensemble de Tiermas plutôt qu'au site de Leire ${ }^{22}$, où existe aussi une source (de caractère non médicinal semble-t-il mais à la vertu miraculeuse d'après une tradition locale signalée à $M$. J. Peréx ${ }^{23}$ ). Car à elle seule la dédicace aux Nymphes ne permet pas de trancher: en effet elle émane d'un individu qui fait figurer sa profession d'aquilegus ${ }^{24}$, ce qui semble indiquer que le voeu aux divinités des eaux concernait plutôt la réussite de son travail qu'une guérison. Si l'autel de Quintus Licinius Fuscus procède bien de Tiermas, la venue de ce technicien étranger à la région ${ }^{25}$ pourraît s'expliquer par des travaux destinés au captage de la source, lors d'un aménagement ou réaménagement des installations sous l'Empire (dont date l'inscription ${ }^{26}$ ).

${ }^{21}$ Le musée possède donc de nombreuses inscriptions (milliaires, stèles funéraires ou autels) originaires de la région des Cinco Villas dans la province de Saragosse dont dépend Baños de Tiermas, situé à la limite de la province de Pampelune. Au Moyen-Age la zone fut très disputée entre l'Aragon et la Navarre, après avoir servi de frontière avec les musulmans: ces derniers s'étaient emparés du château de Ruesta, en face de Baños de Tiermas, mais n'avaient pu passer sur la rive droite du río Aragón. Dans l'Antiquité, la zone semble avoir également constitué une limite entre les territoires del Jacetani au nord, des Suessetani à l'est et des Vascones à l'ouest, avant l'expansion de ces derniers aux dépens des deux autres peuples. Etat de la question et bibliographie dans Perex, M. J., op. cit., págs. $63-69$.

${ }^{22}$ Hypothèse retenue par tous les auteurs qui l'ont étudiée et notamment: TARACENA, $B$. \& VAzQuez DE PARGA, L., «Excavaciones en Navarra. V. La romanización», Príncipe de Viana, 7, 1946, págs. 415-569 (inscription n. ${ }^{\circ} 34$ ); Castillo, C., Gomez Pantoja, J. \& Mauleon, M. D., Inscripciones romanas del Museo de Navarra. Pamplona 1981, n. ${ }^{\circ}$ 26, págs. 52-53.

${ }^{23}$ Ce qui expliquerait l'implantation à cet endroit du monastère (cité dès le milieu du ${ } X^{\circ}$ siècle par Saint Euloge de Cordoue).

${ }^{24}$ Cette fonction (aquilex ou aquilegus) apparaît également sur une inscription de la province de León, à Calda de Boñar: CIL 2, $2964=5726$; ILER 656. Hispania Epigraphica, 1, 1989 signale la nouvelle lecture de F. Diego, Inscripciones romanas de la provincia de León. León 1986, n. ${ }^{\circ} 60$, págs, 76-77. II s'agit bien d'une source thermale, dont l'origine romaine avait déjà été signatèe (comme d'ailleurs celle des sites du nord de l'Hispanie) par Alexandre de Laborde dans son Voyage pittoresque et historique de l'Espagne (1798-1801). Paris 1806-1820, 4 vol.

${ }_{25}$ Vareia (Varea, faubourg de Logroño) était à l'époque de Sertorius une puissante cité des Berones, qui s'opposèrent à lui (Tite-Live, frag. 91) et dont Strabon $(3,4,12)$ rappelle l'origine celtique. II mentionne également le passage de l'Ebre à la hauteur de la ville, Vareia oppido selon Pline $(3,21)$. Le nom de Vareia figure encore chez Ptolémée $(2,6,55)$ et dans l'Itinéraire d'Antonin $(393,2)$ mais l'inscription de Leire est le premier témoignage épigraphique de la cité bérone. Voir VILLACAMPA RUBiO, M. A., Los Berones según las fuentes escritas. Logroño 1980, págs. 44-50.

${ }^{26}$ D'après, M. A. Villacampa Rubio, op. cit., pág. 46, elle ne serait pas antérieure au $\|^{\mathrm{e}}$ siècle de notre ère, et même aux Sévères selon ELORzA, J. C., «Religiones primitivas del País vasco-navarro en época romana", Estudios de Deusto, 20, 1972, págs. 357-366. 
Sources médicinales et thermalisme dans le bassin de l'Ebre ...

Alors que le texte d'Aquae Bilibilitanorum suggère un fonctionnement indigène du site thermal, à Tiermas ce caractère est indécelable dans la documentation actuelle, qui révèle un contexte purement romain: ici le nom des Nymphes n'est pas suivi d'une de ces épithètes qui masquent les divinités locales, l'aquilegus présente une anthroponymie entièrement latine et son origo renvoie à une cité romanisée de la vallée de l'Ebre ${ }^{27}$. Le témoignage épigraphique - si sa localisation est exacte- vient donc seulement confirmer que l'ensemble thermal de Tiermas a une origine au moins romaine, comme l'indique l'archéologie.

Nous ne disposons que de cette dernière pour vérifier l'existence du site de Fitero sous l'Empire. Trois kilomètres à l'ouest de la ville moderne, au lieu dit Baños de Fitero sur la rive gauche du río Alhama, jaillit une eau thermale $\left(46-47^{\circ} \mathrm{C}\right.$.) à dominante chloruro-sodique mais également sulfato-calcique; ses deux sources (de 10 et 20 litres par seconde) furent successivement mises à profit par des établissements thermaux, appelés pour cette raison baños viejos et baños nuevos et situés à un kilomètre de distance.

Bien que l'un et l'autre ne remontent qu'au $X x^{\theta}$ siècle, l'utilisation antérieure du site (d'ailleurs suggérée par la toponymie et l'hydronomie locales) est attestée depuis le Moyen-Age par une documentation écrite. Ces textes font aussi allusion à des bâtiments romains, notamment à un balneum de Caracalla ${ }^{28}$ cité dans le cartulaire de 1157 . En effet, la construction des thermes modernes entraîna la découverte, au milieu du siècle dernier, d'un abondant matériel antique à proximité des baños viejos (lampes, monnaies, céramiques) et de structures non identifiées. Sans avoir retrouvé les thermes proprement dits, on avait déjà reconnu le caractère romain des aménagements hydrauliques réalisés à Baños de Fitero. Ainsi la galerie de capture de la source, décrite en 1930 par le médécin du balnéaire ${ }^{29}$ : haute de $1,75 \mathrm{~m}$ et large de $78 \mathrm{~cm}$, elle avait été

\footnotetext{
${ }^{27}$ Nous ignorons le statut de Vareia, que Pline ne nomme pas parmi les cités ou peuples du conventus caesaraugustanus. Quintus Licinius Fuscus lui porte les tria nomina et n'est donc pas un affranchi (comme de nombreux techniciens des eaux). Mais, si son surnom est bien Fuscus, il pourrait avoir une ascendance servile. Voir KaJANTO, I., The Latin cognomina. Helsinki 1965, pág. 65.

${ }^{28}$ Medrano Marquez, M. M. \& Diaz Sanz. A. M., "Las instalaciones balnearias romanas de Fitero", Primer Congreso general de Historia de Navarra (Pamplona, 1986). Pamplona 1987, t. 2, págs. 491-501. Mezquiriz IRujo, M. A., "Las termas romanas de Fitero", Homenaje a J. M. Lacarra, Príncipe de Viana, Anejo 3. Pamplona 1986, t. 2, págs. 539-554. L'auteur rappelle (pág. 548) qu'il existait, entre les deux établissements modernes, une troisième source thermale, utilisée pour les animaux.

${ }^{29}$ Mezquiriz lrujo, M. A., op. cit., pág. 542.
} 
taillée au pic sur $65,44 \mathrm{~m}$ de long à partir de la source thermale, qui jaillit des parois et du dol d'une cavité aménagée en rotonde.

L'ampleur de tels aménagements laissait supposer des installations romaines d'une certaine importance: de fait elles comprenaient au moins quatorze bassins circulaires, apparus fortuitement en 1982 dans les baños viejos à l'occasion de travaux qui ont confirmé la superposition des établissements ancien et moderne. Malheureusement, seul un exemplaire a pu être conservé (car il se trouvait dans une zone d'accès aux thermes modernes) et demeure visible aujourd'hui.

II s'agit ${ }^{30}$ d'une structure circulaire (large de $2 \mathrm{~m}$ et profonde de $1 \mathrm{~m}$ ) qui présente, à $25 \mathrm{~cm}$ et $50 \mathrm{~cm}$ du fond, deux banquettes concentriques au diamètre décroissant $(1,55 \mathrm{~m}$ et $1,04 \mathrm{~m})$. Les pierres en opus quadratum (de module romain) avaient été taillées en voussoirs, et soigneusement appareiliées comme celles du fond. Peut-être un placage de marbre ${ }^{31}$ (dont on a rencontré des fragments à proximité) recouvrait-il les blocs du bassin. Celui-ci ne disposait pas d'orifice pour évacuer l'eau, qui arrivait par un canal convexe creusé dans la banquette supérieure: la cavité (large de $17 \mathrm{~cm}$ et profonde de $7 \mathrm{~cm}$ ) est visible sur une longueur de $51 \mathrm{~cm}$ en direction du château d'eau, situé à quelques mètres de là.

Cet édifice romain sert toujours à répartir l'eau (venant de la galerie qui a été percée à la hauteur du bâtiment pour accroître le débit) entre les différentes salles des thermes modernes. De forme rectangulaire, il est conservé sur 1,60 $\mathrm{m}$ de hauteur et comporte trois assises (la hauteur de la dernière représentant le double de celle des précédentes) couronnées d'une corniche. Les blocs, assemblés à joints vifs et présentant des traces de bossage, ont des dimensions très variables. A l'une des extrémités, un arc $(1,25 \mathrm{~m}$ de haut, $0,91 \mathrm{~m}$ de large à la base et $0,73 \mathrm{~m}$ au sommet) donne accès à l'intérieur du bâtiment et devait servir au nettoyage, rendu indispensable par la décantation de l'eau mais aussi par d'importants dépôts de sel. On voit, sur la paroi interne proche de l'arc, une ouverture triangulaire (de $21 \mathrm{~cm}$ de large et $24 \mathrm{~cm}$ de haut) par laquelle l'eau allait alimenter les bassins antiques. Un fragment de canali-

${ }^{30}$ Pour la description détaillée des vestiges de Baños de Fitero, voir Mezouiriz Irujo, $M$. A., op. cit., pág. 546 et Medrano Marquez, M. M. \& Diaz Sanz, A. M., op. cit., págs. 491-493.

${ }^{31}$ Comme à Néris-les-Bains en Gaule, où les thermes d'Aquae Neri comportaient notamment une piscine entourée de trois gradins, en pierre de taille et placage de marbre. Voir CoRrocher, J., "Sources et installations thermales en Bourbonnais", Les eaux thermales et les cultes des eaux..., op. cit., pág. 179. 
sation en céramique ${ }^{32}$ de $18 \mathrm{~cm}$ de diamètre a d'ailleurs été retrouvé dans le périmètre des thermes modernes.

Comme l'eau de la source atteint près de $50^{\circ} \mathrm{C}$. dans le castellum aquae, son passage dans l'édifice entraînait un abaissement naturel de sa température avant qu'elle n'arrive dans les quatorze bassins. Ceux qui étaient les plus proches du château d'eau pouvaient d'ailleurs servir aussi bien de laconicum que de caldarium, et notamment l'exemplaire conservé: ses trois degrés permettaient soit de prendre un bain de vapeur en s'asseyant sur le bord du bassin, soit de se plonger plus ou moins profondément dans l'eau thermale selon les parties du corps à soigner ${ }^{33}$.

Aucun matériel antique n'ayant été signalé lors de la mise au jour des bassins ou recueilli dans le château d'eau, c'est d'après les techniques de construction (bossages, opus quadratum) des structures conservées que l'on a daté ${ }^{34}$ l'ensemble thermal de fitero des $I^{e}-1 l^{e}$ siècles de notre ère. Nous ne savons pas s'il fonctionnait toujours au Bas-Empire ni s'il existait déjà sous la République. Mais la proximité de Gracchuris (Alfaro), fondée en 179 par Tibérius Sempronius Grachus et qu'une route reliait à Numantia par la vallée du río Alhama, ainsi que celle de Turiaso (Tarazona de Aragón) inciteraient à penser que le site thermal a pu se développer assez tôt, grâce à la fin des guerres celtibères et à la romanisation de la vallée de l'Ebre sous Sertorius.

En l'absence de tout témoignage épigraphique, on ignore pour le moment quelles étaient les divinités honorées à Fitero et s'il s'agissait de figures classiques ou indigènes. Remarquons enfin que -comme à Tiermas- on a affaire à un ensemble rural et situé près d'une frontière, antique puis moderne: en effet Baños de Fitero est à la limite des royaumes médiévaux de Castille, d'Aragon et de Navarre (ce que rappelle le mot de «fitero»), mais également à l'extrémité méridionale de la province de Pampelune: cette enclave perpétue l'avancée du territoire des Vascones sur la rive droite de l'Ebre ${ }^{35}$, où ils possédaient les cités de Gracchuris (autrefois celtibère) et de Cascantum (Cascante), située en face de la Turiaso celtibère.

\footnotetext{
${ }^{32}$ Vitruve décrit (en 8,6 ) les avantages de ces conduits emboîtés les uns dans les autres, qui étaient en particulier plus faciles à fabriquer et moins onéreux que les tuyaux en plomb. Voir à ce sujet AdAM, J. P., La construction romaine. Paris 1984, pág. 276.

${ }^{33}$ Les médecins antiques conseillaient déjà de ne traiter que la seule zone malade, pratique particulièrement bien adaptée aux affections rhumatismales et circulatoires pour lesquelles les eaux de Fitero sont recommandées.

${ }^{34}$ Medrano Marquez, M. M. \& Diaz Sanz, A. M., op. cit, pág. 494.

${ }^{35}$ Peréx, M. J., op. cit., págs. 63-69.
} 
Quant à Panticosa, il ne figure que depuis peu sur la liste des lieux antiques de thermalisme attestés dans le bassin de l'Ebre. Ce village pyrénéen, d'altitude élevée (1636 $\mathrm{m}$ ) et d'accès difficile, utilisait jusqu'à ces dernières années cinq sources sulfhydriques qui jaillissent à $27^{\circ} \mathrm{C}$. et alimentent l'établissement actuel. Mais on ne disposait pas - comme pour les trois autres sites thermaux - de documents, de textes, ni même d'une tradition locale faisant remonter son existence jusqu'à l'Antiquité.

En 1951 on trouva à proximité d'une des sources un as augustéen ${ }^{36}$ frappé à Casaraugusta, ce qui montrait que Panticosa était déjà connu au début de l'Empire, et fréquenté malgré l'isolement du site. Mais c'est la découverte fortuite d'une autre source, près de la chapelle édifiée au fond du cirque montagneux, qui apporta la preuve du fonctionnement antique de Panticosa. L'eau, qui jaillit du massif granitique à une température ${ }^{37}$ de $51^{\circ} \mathrm{C}$. (donc nettement plus élevée que celle des autres sources), est fortement sulfurée et radio-active, propriétés qui furent appréciées au moins dès la période romaine.

En effet les travaux modernes destinés au captage de la nouvelle source révèlèrent à $4,20 \mathrm{~m}$ de profondeur une ouverture taillée dans la roche et permettant d'atteindre directement le niveau de l'eau thermale. Le puits antique avait une section carrée, que délimitaient quatre madriers en chêne ${ }^{38}$ longs de 62 à $75 \mathrm{~cm}$ et emboîtés l'un dans l'autre par des mortaises de $6 \mathrm{~cm}$. Sur le ciment hydraulique naturel dans lequel il avait été foré reposaient plusieurs monnaies, très corrodées par le milieu ambiant: on a pu néanmoins identifier un semis, un quadrans, ainsi que deux as, l'un de Sagonte et l'autre frappé sous Tibère par la colonie romaine de Celsa (Velilla del Ebro, en aval de Saragosse).

La prèsence de ces pièces a fait penser qu'ils s'agissait d'ex-voto ${ }^{39}$. Elles témoignent en tout cas de la fréquentation de la source thermale au $1^{\text {er }}$ siècle de notre ère, alors que nous ignorons tout de son fonctionnement avant ou après cette date. La structure du puits pourrait suggérer un pré-

${ }^{36}$ Beltran, A., "Moneda romana de Zaragoza, hallada en Panticosa", Caesaraugusta, 4, 1953, págs. 129-140.

37 Beltran, A., «Los hallazgos del balneario de Panticosa (Huesca)», Caesaraugusta, 5 , 1954, págs. 196-200. Selon l'auteur, c'est à cette source que renverrait le toponyme "Caldarés», jusqu'ici inexpliqué, Lostal Pros, J., «Arqueología del Aragón romano», Caesaraugusta, 41-42, 1977, pág. 9-10.

38 Beltran, A., op. cit., pág. 199, raproche ce type de puits en bois des exemplaires retrouvés dans les campements militaires du limes rhénan, ainsi que de ceux du site médicinal des Fontaines-Salées près de Velezay.

39 Lostal Pros, J., op. cit., pág. 10: «... las monedas halladas corresponden sin duda a votos hechos por enfermos curados de sus dolencias...». 
Sources médicinales et thermalisme dans le bassin de l'Ebre ...

cédent indigène ${ }^{40}$ mais on sait cette technique a aussi été employée à l'époque romaine pour les sources médicinales, bien qu'elle ait laissé peu de traces en raison du caractère périssable du matériau utilisé.

Les structures recueillies à ce jour sont trop sommaires pour qu'on puisse parler d'établissement thermal. Prélevait-on l'eau directement dans le puits, ou celui-ci était-il raccordé à d'autres installations? Rien ne permet de le dire actuellement, ni de savoir quelle ampleur avait le site qui, malgré son altitude élevée et son accès difficile, n'était cependant pas aussi isolé qu'il le paraît: en effet il communiquait avec la vallée de Tena où une voie romaine ${ }^{41}$ suivait le cours du río Gallego.

L'exemple de Panticosa amène à réfléchir sur les problèmes de la documentation disponible et permet d'envisager plusieurs axes de recherche afin de complèter nos connaissances actuelles.

Tout d'abord, on constate que les sites thermaux antiques abondent sur le versant français des Pyrénées, alors que Panticosa est le seul attesté sur le versant espagnol où existent pourtant des établissements modernes. Ces derniers ont-ils une origine antique, comme c'est le cas des installations de Alhama de Aragón, Tiermas, Fitero et de Panticosa (dont on ignorait l'ancienneté jusqu'à ces denières années)? Pour le vérifier, il serait intéressant de disposer d'une étude historique et toponymique sur les thermes des Pyrénées espagnoles ${ }^{42}$ et d'y mener des prospections ou des fouilles archéologiques, facilitées à l'heure actuelle par la fermeture de plusieurs d'entre eux.

Une telle recherche a été entreprise dans la partie occidentale du bassin de l'Ebre ${ }^{43}$ où l'on est frappé par le fait que, souf un, tous les ensembles thermaux modernes se trouvent sur l'ancien territoire vascon: non seulement dans le saltus Vasconum (Pyrénées et Cordillère Cantabrique) mais dans l'ager Vasconum (autour de Pampelune) ainsi que sur la rive droite de l'Ebre, où l'on en compte quatre non loin de Baños de Fitero. Tous ces sites du Système Ibérique fonctionnèrent'ils eux-aussi durant

${ }^{40}$ Lostal Pros, J., ibid. Remarquons que le cours du rio Gallego (comme celui de l'Aragón dans la zone de Tiermas) fut une voie de pénétration celtique, ce dont témoigne la toponymie ancienne et moderne: Gallicus flumen, Gallicum, Berdún, Navardún, etc.

${ }^{41}$ On en a retrouvé des vestiges à Biescas. Voir Dominguez Arranz, A., Magallon Botaya, M. A. \& Casado Lopez, P., Carta arqueológica de España. Huesca. Huesca 1984, pág. 65.

${ }^{42}$ Ainsi Bénasque, Caldas de Bohí, Las Vilas del Turbó, à l'est de Panticosa.

${ }^{43}$ Voir, dans ce même volume, la communication de M. J. PERÉx \& M. UNZU, "Termalismo y habitat en el valle medio del Ebro en época antigua». 
l'Antiquité, et en mème temps? Et qu'en était-il de Paracuellos de Jiloca, proche de Bilbilis et d'Aquae Bilbilitanorum? II est possible que, parmi des sources voisines, on ait exploité les unes et pas les autres, ou que certaines aient périclité à un moment donné, surtout si elles avaient des propriétés identiques (faciles à vérifier, s'agissant d'eaux analysés). A chaque fois il convient de préciser le rapport des sites aux routes, aux territoires des peuples et aux villes antiques car il joua un rôle important, à en juger par l'exemple des établissements dont nous connaissons l'origine romaine.

Ensuite, constatons qu'on ne dispose pas pour Panticosa (ni d'ailleurs pour Fitero) de documentation épigraphique associée au site thermal. Sur les dix inscriptions [fig. 2] du bassin de l'Ebre qui semblent ${ }^{44}$ appartenir au monde de l'eau, seules deux d'entre elles se rapportent à des installations thermales. Les autres présentent des difficultés parce que, selon les cas, on ignore leur provenance exacte (Ujué), leur lien avec un point d'eau (Tricio et Rasillo de Cameros), la nature du site (Cabriana) ou le caractère de la source (Araya et Valtierra).

$\mathrm{Si}$ les deux premiers problèmes paraissent sans solution (sauf nouveaux renseignements sur les circonstances dans lesquelles l'inscription fut découverte), on peut rémédier aux deux derniers par une prospection à la fois archéologique et géologique, afin de connaître la nature des installations et les propriétés de la source. Mais il convient de rester prudent dans l'interprétation des données.

En effet, la présence d'un habitat à proximité d'une source médicinale ne signifie pas que celle-ci a été utilisée à des fins thérapeutiques: ainsi pour les eaux sodées, dont on a pu n'exploiter que le sel (notamment en milieu pastoral). Par ailleurs, une pratique votive réelle a parfois concerné des eaux sans vertu curative ${ }^{45}$, alors qu'inversement une dédicace à une

${ }^{44}$ Le caractère «aquatique» de Lacubegi et surtout de Varna/Uvarna n'est pas accepté par tous les auteurs. Bibliographie complémentaire: ABAsolo, J. C. \& ElorzA, J. C., «Nuevos teónimos de época romana en el Pais Vasco-Navarro", Est. de Arqueología Alavesa, 6, 1974, pág. 247-258. Albertos, M. L., “Alava prerromana y romana. Estudio linguístico», Est. de Arqueología Alavesa, 4, págs. 107-233. Crespo Ortiz, S., "Aspectos de la romanización de Álava. Prosografia y sociedad", Est. de Arqueología Alavesa, 10, págs. 241-296. ElorzA, J. C., «Ensayo topográfico de epigrafía romana en Alava», Est. de Arqueologia Alavesa, 2, 1967, págs. 119-185. ElorzA, J. C., "A propósito de algunas divinidades de época romana en Álava», Cong. Nac. Arq. 11 (1969). Zaragoza 1970, págs. 815-820, Elorza, J. C., Albertos, M. L., \& Gonzalez, A., Inscripciones romanas en la Rioja. Logroño 1980. Espinosa Ruiz, U., Epigrafia romana de la Rioja. Logroño 1986. FAtAs, G. \& MARTin Bueno, M. A., Epigrafía romana de Zaragoza y su provincia. Zaragoza 1980. Ruiz, S., "Un ara votiva en Comuniòn», Est. de Arqueología Alavesa, 6, 1981, págs. 297-305.

${ }^{45}$ C'est le cas par exemple de la source de la Seine, où l'on a trouvé de nombreux exvoto en bois. 
divinité des eaux (médicinales ou non) n'implique pas que le voeu eit été accompli à la suite d'une guérison (on l'a vu avec l'inscription de Leire/ Tiermas) ni qu'il s'agisse de divinités guérisseuses: sur ce plan, l'exemple des Nymphes est à la fois le plus fréquent et le plus ambigu. Une approche terminologique et méthodologique s'avère donc indispensable pour déterminer la véritable nature des sites, des inscriptions et des structures antiques se rapportant au domaine de l'eau.

\section{RESUMEN}

Sólo se conocen cuatro yacimientos termales antiguos en la cuenca del Ebro, lo que parece muy poco ya que cubre la sexta parte de España y contiene numerosas fuentes medicinales. Algunas de las que están cercanas a poblados antiguos pueden haber sido utilizadas desde la época romana - si no prerromana-; pero, como casi siempre faltan los textos, sólo podemos identificar los establecimientos termales gracias a la arqueología, epigrafia o numismática. Tal documentación resulta desigual y difícil de utilizar, como veremos para los cuatro yacimientos atestiguados: Alhama de Aragón, Tiermas, Fitero y Panticosa.

\section{ABSTRACT}

Only four ancient water-cure sites are known in the Ebro basin, a very poor number considering to its area (the sixth part of Spain) and the many medicinal sources it conceals. Several of those are close to ancient habitats may have been in use since Roman -if not preroman- times; however, as texts are generally lacking, we can't identify water-cure establishments but by archaelogy, epigraphy or numismatics. Such documentation is unequal and hard to exploit, as will be seen with the four testified sites: Alhama de Aragón, Tiermas, Fitero and Panticosa. 


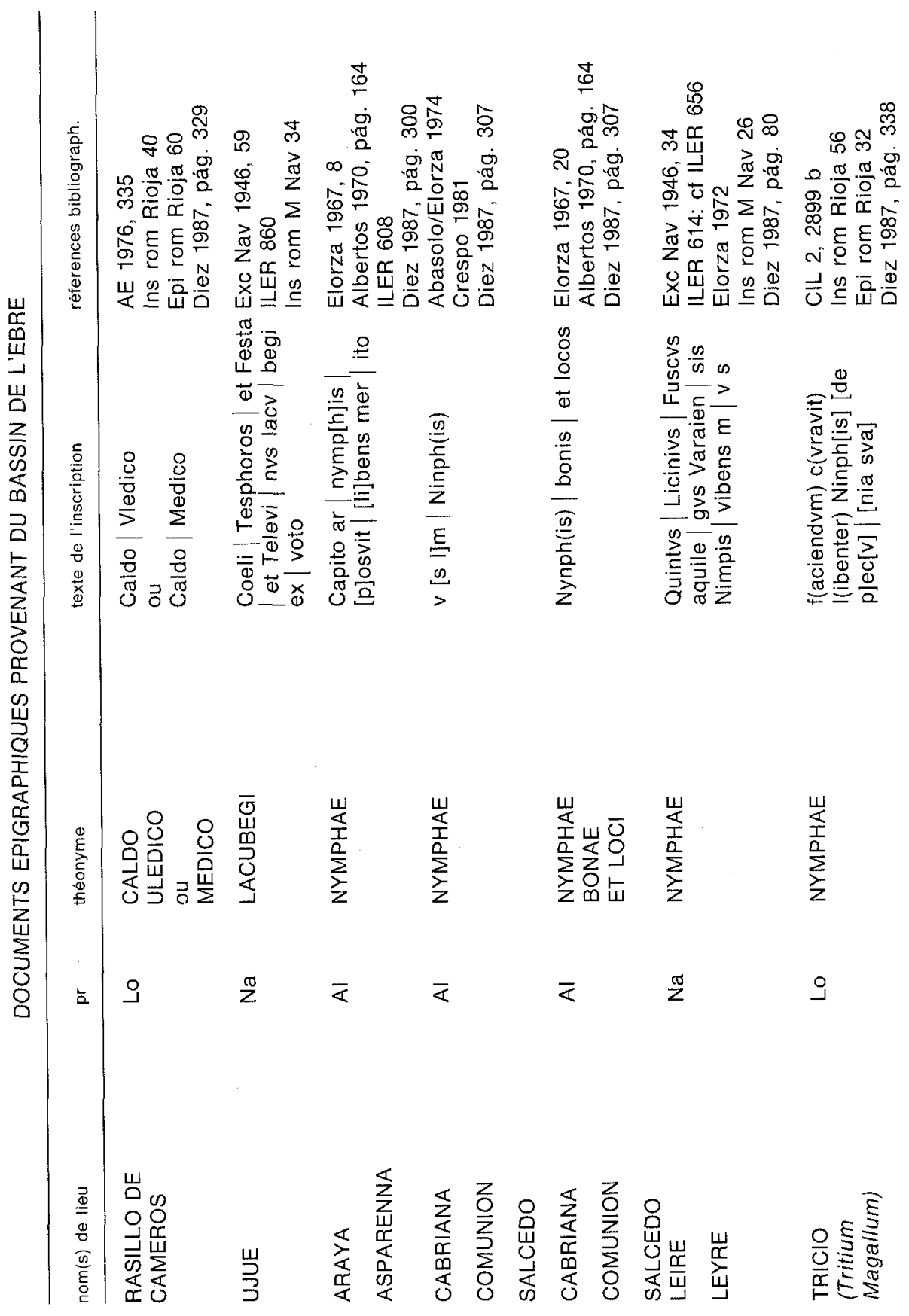




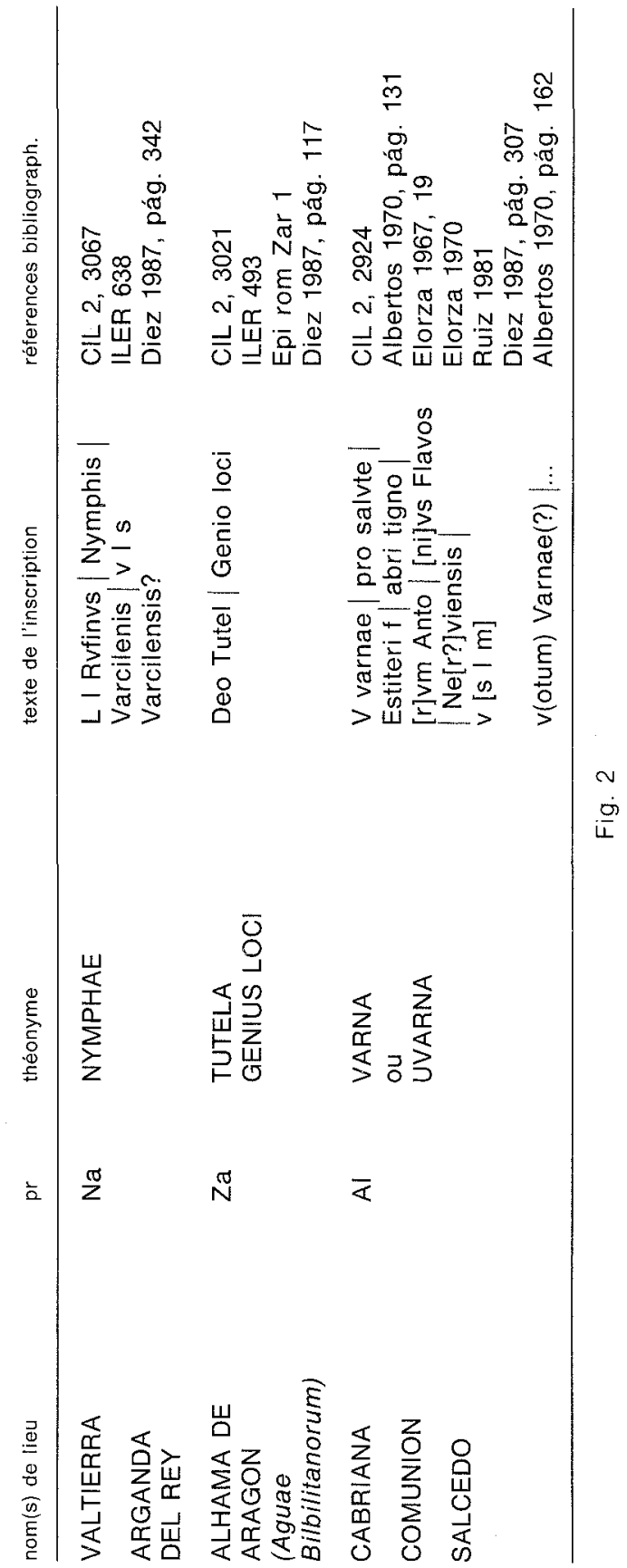


